\title{
STRUCTURAL PROPERTIES OF HYDROGEN PLASMA
}

\section{Tomiris Ismagambetova ${ }^{1}$, Maratbek Gabdullin ${ }^{2}$, Tlekkabul Ramazanov ${ }^{3}$}

\begin{abstract}
This paper considers hydrogen, non-ideal plasma. The structural properties of such plasma were investigated. To study properties of plasma, effective potentials describing the interaction between particles were used. These potentials take into account various effects: screening and quantum-mechanical (diffraction and symmetry). The Pauli exclusion principle prohibits the simultaneous presence of two identical particles with a half-integer spin (in this case, electrons) in the same state. Pair correlation functions were calculated in hyper-netted chain approximation for the integral equation of the OrnsteinZernike on the basis of the interaction potentials. The symmetry effect is more pronounced at short distances and for higher values of density. The antiparallel direction of the electron spins increases the probability of finding electrons at distance $\mathrm{R}$ from each other, the parallel direction decreases this probability due to the prohibition of the presence of two electrons with the same spins in the same state.
\end{abstract}

UDC Classification: 533.9; DOI: http://dx.doi.org/10.12955/cbup.v6.1300

Keywords: plasma, potential, structural.

\section{Introduction}

In this work, non-ideal hydrogen plasma was considered. Non-ideal plasma is plasma in which interparticle interactions have a major effect, that is, the average energy of interaction becomes comparable to the kinetic energy. The properties of such plasma can be calculated using a model of interaction between the particles in the plasma. The model of interparticle interactions that was used takes into account the collective effects at large distances and the quantum-mechanical effects at small distances.

\section{Method}

Pseudopotential interactions used in this paper take into account the screening effects at large distances and the quantum-mechanical effects of diffraction - at small distances. These potentials were obtained in the work by Ramazanov and Dzhumagulova (2002), Ramazanov, Dzhumagulova, \&Gabdullin (2010):

$$
U_{a b}(r)=\frac{Z_{a} Z_{b} e^{2}}{\sqrt{1-4 \lambda_{a b}^{2} / r_{D}^{2}}}\left(\frac{e^{-B r}}{r}-\frac{e^{-A r}}{r}\right),
$$

where $A^{2}=\frac{1}{2 \lambda^{2}}\left(1+\sqrt{1-\lambda_{a b}^{2} / r_{D}^{2}}\right), B^{2}=\frac{1}{2 \lambda^{2}}\left(1-\sqrt{1-\lambda_{a b}^{2} / r_{D}^{2}}\right), Z_{a} e, Z_{b} e$ are the atomic numbers of a, b particles, $\lambda_{a b}=\hbar / \sqrt{2 \pi m_{a b} k_{B} T}$ is the thermal de-Broglie wave length, $m_{a b}=m_{a} m_{b} /\left(m_{a}+m_{b}\right)$ is the reduced mass, $r_{D}=\left(k_{B} T /\left(4 \pi e^{2} \sum_{j} n_{j} Z_{j}^{2}\right)\right)^{1 / 2}$ is the Debye radius, also dimensionless parameters: $\Gamma=\frac{(Z e)^{2}}{a k_{B} T}$ is coupling parameter, $r_{s}=a / a_{B}$ is density parameter, $a=\left(3 /\left(4 \pi n_{e}\right)\right)^{1 / 3}$ is the average distance between electrons, $a_{B}$ is the Bohr radius.

To take into account the quantum-mechanical effect of symmetry the following formula was used for different electron spin directions ( $\mathrm{S}=0$ - antiparallel spins, $\mathrm{S}=1$ - parallel spins):

$$
U_{e e, 0}^{S(T)}(r)=-k_{B} T \ln \left(1 \pm \exp \left(-\frac{r^{2}}{\lambda_{e e}^{2}}\right)\right),
$$

and for average direction of electron spins:

\footnotetext{
${ }^{1}$ Institute of Experimental and Theoretical Physics, al-Farabi Kazakh National University, Kazakhstan, miriael@mail.ru

${ }^{2}$ Kazakh British Technical University, Kazakhstan, gabdullin@physics.kz.

${ }^{3}$ Institute of Experimental and Theoretical Physics, al-Farabi Kazakh National University, Kazakhstan, tlekkabul.ramazanov@kaznu.kz
} 


$$
U_{a b}(r)=-\delta_{a e} \delta_{b e} k_{B} T \ln \left(1-\frac{1}{2} \exp \left(-\frac{r^{2}}{\lambda_{e e}^{2}}\right)\right) .
$$

The hyper-netted chain approximation (HNC) of the integral equation of Ornstein-Zernike from work by Goodstein (2002) was used to calculate pair correlation functions:

$$
\begin{gathered}
h(\vec{r})=C(\vec{r})+n \int C\left(\vec{r}_{3}-\vec{r}_{1}\right) h\left(\left|\vec{r}_{3}-\vec{r}_{2}\right|\right) d r_{3}, \\
C_{H C A}(r)=h(r)-\ln g(r)-\frac{U(r)}{k_{B} T},
\end{gathered}
$$

where $h(\vec{r})=g(\vec{r})-1$ is the full correlation function, $C(\vec{r})$ is the direct correlation function, and $U(r)$ is the interaction potential.

\section{Results}

The pair correlation functions for different interaction potentials are presented in Figure 1. Black and red lines show the results on the basis of Debye and Deutsch potentials, green and blue lines - the results based on the potential (1) without and with the symmetry effect.

Pair correlation functions for different directions of spins are shown in Figure 2. Black line represents the results based on the potentials (1) and (2) for antiparallel spins and green line - the results for parallel spins, red line - the results based on the potentials (1) and (3) for average direction of the electron spins.

Figures 3-4 show pair correlation functions on the basis of potentials (1) and (2) for antiparallel (dashed lines) and parallel (dash-dotted lines) directions of spins, and for potentials (1) and (3) for average direction (solid lines). The results in Figure 3, denoted by lines of different colors, represent data for $\Gamma=0.1,0.2,0.3,0.5$. The results in Figure 4, also indicated by lines of different colors, represent data for $r_{s}=1,2,5$.

Figure 1: Pair correlation functions for electron-electron interaction at $\mathrm{r}_{\mathrm{S}}=5, \Gamma=0.2$

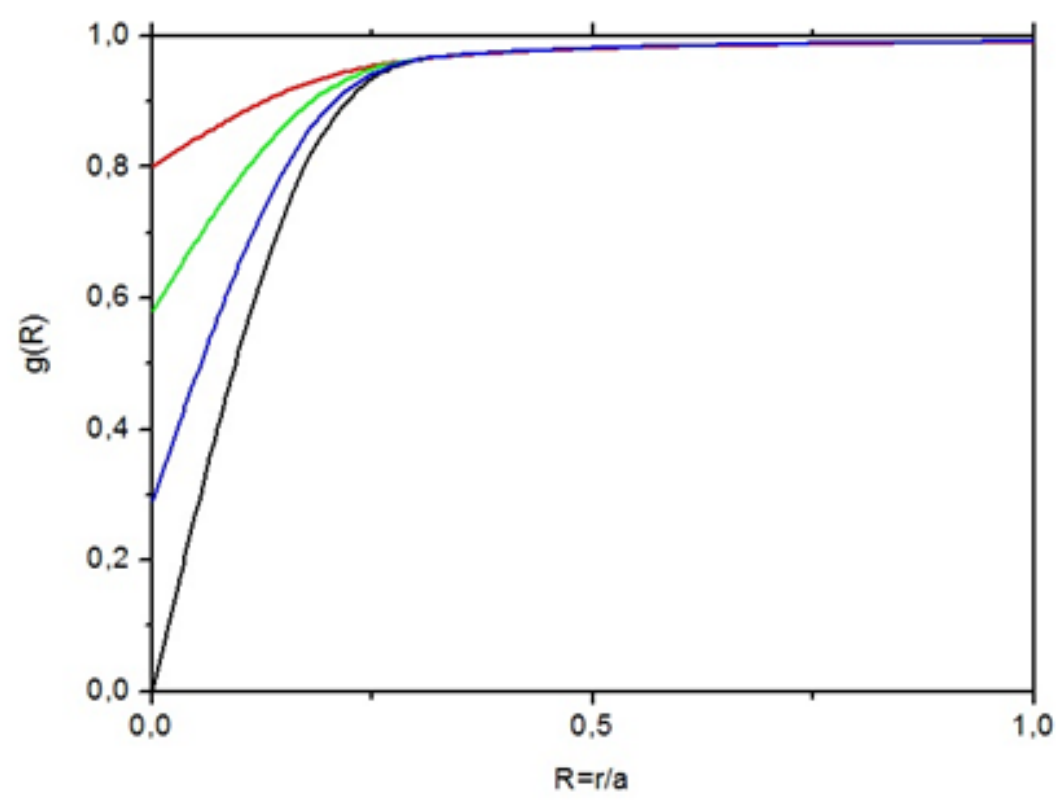

Black line is the results for Debye potential, red line - Deutsch potential, green line - potential (1), blue line - potential (1) with the addition of (3)

Source: Authors 
Figure 2: Pair correlation functions for electron-electron interaction at $\mathrm{r}_{\mathrm{S}}=1, \Gamma=0.2$

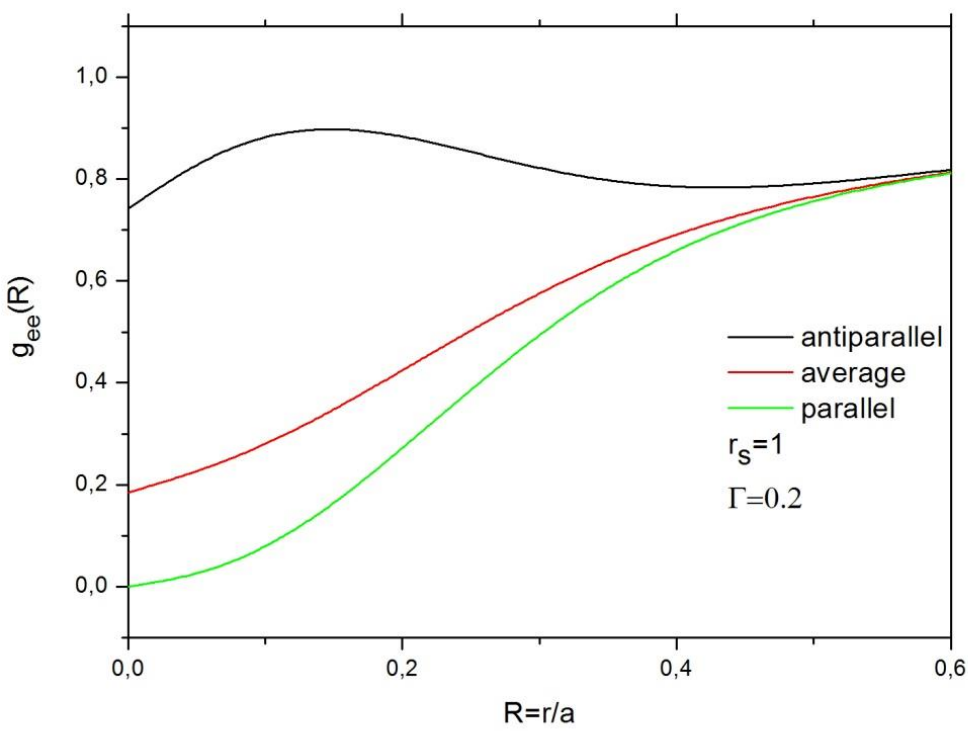

Black line is the results for the potential (1) with the addition of (2) for the antiparallel direction of the electron spins, red line - for the potential (1) with the addition of (3) for the average direction of the electron spins, green line - for the potential (1) with the addition of (2) for the parallel direction of the electron spins

Source: Authors

Figure 3: Pair correlation functions for electron-electron interaction at $r_{S}=1, \Gamma=0.1,0.2,0.3,0.5$

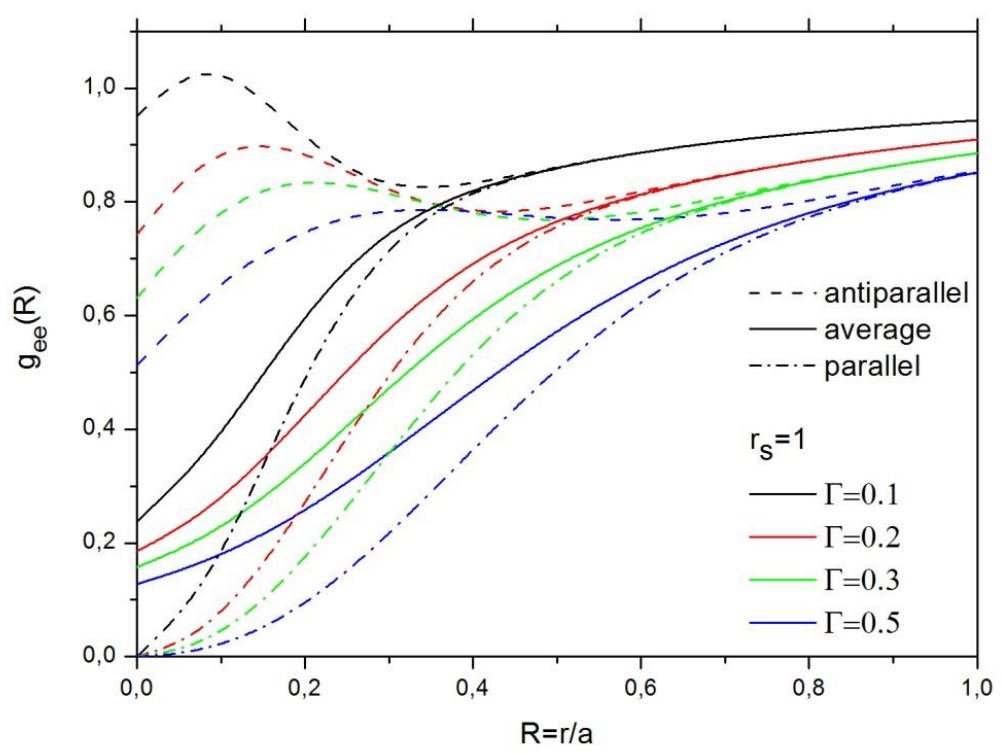

Dashed line is the results for the potential (1) with the addition of (2) for the antiparallel direction of the electron spins, solid line - for the potential (1) with the addition of (3) for the average direction of the electron spins, dash-dotted line - for the potential (1) with the addition of (2) for the parallel direction of the electron spins

Black line is the results for $\Gamma=0.1$, red line $-\Gamma=0.2$, green line $-\Gamma=0.3$, blue line $-\Gamma=0.5$ 
Figure 4: Pair correlation functions for electron-electron interaction at $\Gamma=0.2, r_{S}=1,2,5$

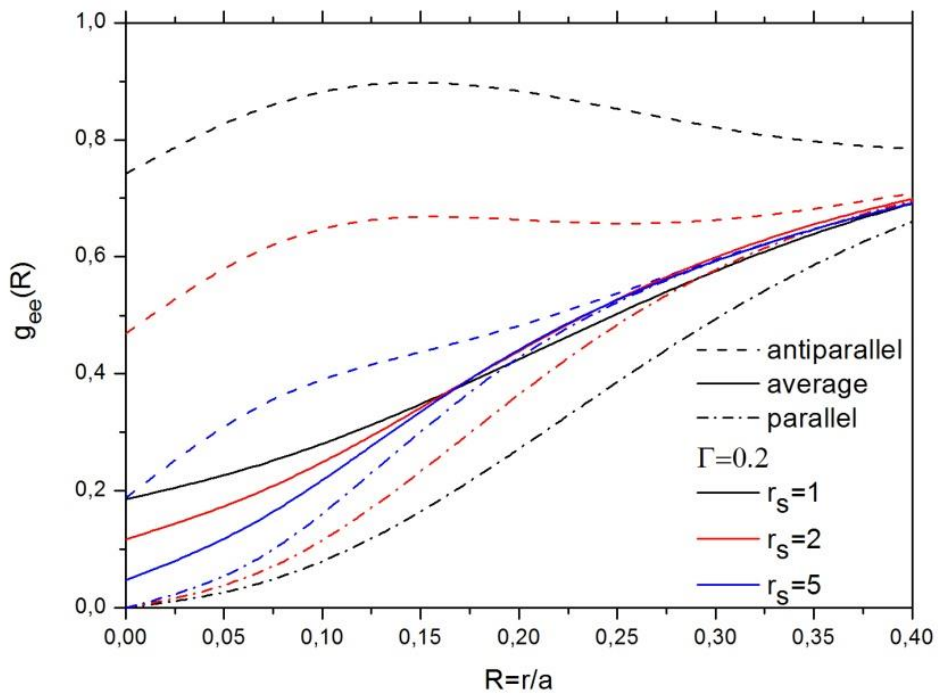

Dashed line is the results for the potential (1) with the addition of (2) for the antiparallel direction of the electron spins, solid line - for the potential (1) with the addition of (3) for the average direction of the electron spins, dash-dotted line - for the potential (1) with the addition of (2) for the parallel direction of the electron spins

Black line is the results for $r_{S}=1$, red line $-r_{S}=2$, blue line $-r_{S}=5$

Source: Author

\section{Conclusion}

The effect of symmetry takes into account the Pauli exclusion principle. The effect of the symmetry effect, as a quantum-mechanical effect, is more pronounced at short distances and for denser plasma. The antiparallel direction of the electron spins increases the probability of finding electrons at distance $\mathrm{R}$ from each other, the parallel direction decreases this probability due to the prohibition of the presence of two electrons with the same spins in the same state.

\section{Acknowledgments}

This work has been supported by the Ministry of Education and Science of Kazakhstan under grant for project №AP05134366.

\section{References}

Goodstein, D.L. (2002) States of Matter. Dover publications, Inc., 207-210.

Ramazanov, T.S., Dzhumagulova, K.N. (2002). Effective screened potentials of strongly coupled semiclassical plasma. Phys. Plasmas, 9 (9), 3758-3761.

Ramazanov, T.S., Dzhumagulova, K N., Gabdullin, M.T. (2010). Effective potentials for ion-ion and charge-atom interactions of dense semiclassical plasma. Phys. Plasmas., 17 (4), 042703. 